\title{
Indian Movie Face Database: A Benchmark for Face Recognition Under Wide Variations
}

\author{
Shankar Setty, Moula Husain, Parisa Beham, Jyothi Gudavalli, Menaka Kandasamy, \\ Radhesyam Vaddi, Vidyagouri Hemadri, J C Karure, Raja Raju, B Rajan, Vijay Kumar and C V Jawahar
}

\begin{abstract}
Recognizing human faces in the wild is emerging as a critically important, and technically challenging computer vision problem. With a few notable exceptions, most previous works in the last several decades have focused on recognizing faces captured in a laboratory setting. However, with the introduction of databases such as LFW and Pubfigs, face recognition community is gradually shifting its focus on much more challenging unconstrained settings. Since its introduction, LFW verification benchmark is getting a lot of attention with various researchers contributing towards state-of-the-results. To further boost the unconstrained face recognition research, we introduce a more challenging Indian Movie Face Database (IMFDB) that has much more variability compared to LFW and Pubfigs. The database consists of 34512 faces of 100 known actors collected from approximately 103 Indian movies. Unlike LFW and Pubfigs which used face detectors to automatically detect the faces from the web collection, faces in IMFDB are detected manually from all the movies. Manual selection of faces from movies resulted in high degree of variability (in scale, pose, expression, illumination, age, occlusion, makeup) which one could ever see in natural world. IMFDB is the first face database that provides a detailed annotation in terms of age, pose, gender, expression, amount of occlusion, for each face which may help other face related applications.
\end{abstract}

\section{INTRODUCTION AND RELATED WORK}

There are many popular databases for benchmarking face recognition algorithms [1]-[5]. Establishing accuracies on these databases has become a mandatory requirement for reporting progress in face recognition. This resulted in systematic progress in this area, specially for laboratory or co-operative environments. After several decades of research, steady performance has been achieved on these databases. For instance, on Yale database [2], it has improved from 58.14\% [6] to $99.3 \%$ [7] in a span of 10 years. To facilitate research in an unconstrained setting, Labeled faces in the wild (LFW) [8] and Pubfigs [9] databases were introduced recently. Images in these databases were harvested from the Internet thereby capturing large variations that are seen in the wild. PubFigs is similar to LFW in terms of variability of faces but contains more images per person on average. Ever since its release, gradual progress is happening in improving LFW verification benchmark due to the effort by various researchers [8].

Our main motivation to introduce a new database is to provide a large set of unconstrained face images overcoming some of the limitations as explained below, of LFW and PubFigs. First, since the images in these databases are collected from

This work was done as part of the Summer research workshop at CVIT, IIITH. S.S and M.H are from BVBCET, Hubli. R.S is from VRS college, P.B is from Vickramce, MDU. M.K is from TCE, R.R is from PSYEC, V.H and J.C.K are from SDM, Dharwad and remaining authors are from CVIT, IIITH.

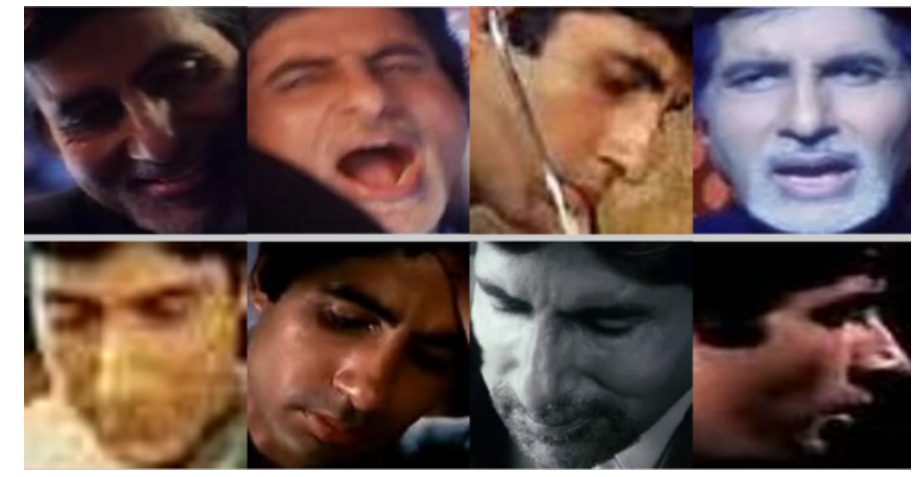

Figure 1. Spectrum of variations in age, pose, expressions, resolution, illumination and amount of occlusion in IMFDB. Examples of Amitabh Bachchan's faces in IMFDB.

Internet sources such as Yahoo news, they mostly contain pose, illumination, resolution variations. However, large fraction of images of a particular person have similar public appearance. Second, since the images are collected from Internet through a search query, they may not cover significant age variations. Third, public figures often retain the identity (appearance, dress patterns, expressions) over sessions reflecting their public behaviour. Fourth, LFW was built on images which are detected by Viola-Jones face detector thereby focusing only on end-to-end systems that have automatic face detection followed by recognition. As mentioned in [8], the use of face detector may result in a subset of possible variations of pose, scale, and occlusion. However, we believe that it is equally important to focus on improving the performance of face recognition algorithms in applications such as identifying criminal suspects or image retrieval from videos where manual detection is feasible/affordable and performance is important. The need for more progress in handling pose, occlusion, resolution, etc is recently stressed upon in a recent case study on unconstrained face recognition [10].

Inspired by LFW, we introduce a challenging database that can critically help unconstrained face recognition research in general, and Indian setting in particular. While being related to these databases in some of the dimensions, we supplement these databases in many ways as explained later. We have kept the following design guidelines in mind while building the database. (i) Capturing images with cameras having different resolutions. (ii) A database of Indian subjects. Most of the previous databases (except for [11]) were built with face images of non-Indians, and the appearance (eg. color and texture) and expressions of the Indian faces could be significantly different from that of these subjects. (iii) Capturing faces in a natural setting (often referred to as "wild"). This results in 
face images with wide variations in pose, illumination and partial occlusions unlike many of the existing databases (eg. Yale, AR) (iv) A database of faces which cover significant age variations for each person. This can help in designing recognition schemes that can robustly recognize individuals independent of the age. This demanded the use of images that were captured over a wide span of years. This is in contrast to the existing databases that are built over a short time period which naturally does not cover age variations. (v) Capturing rich variety of facial expressions as seen in natural world other than pre-defined expressions like smiling, anger, etc. This is often hard to obtain in a laboratory setting. Also, we would like to have the expressions as natural as possible, and therefore capture these expressions from the experts (popular actors). (vii) Cover extreme effects of makeups and facial appearance manipulation that a typical face recognition method will have to address in stress-tests.

We find that faces captured from Indian movies could meet the diverse requirements as listed above. In this work, we present one of the largest face databases (34512 face images of 100 individuals) collected and carefully annotated from approximately 103 Indian movies. We refer this database as Indian Movie Face Database (IMFDB). Specific details of the database are discussed in Section II. In addition to the impact on face recognition research, this database has utility for various related tasks like (i) Face pose estimation (ii) Expression recognition (iii) De-Identification (iv) Photometric and geometric calibration of cameras (v) Image forensics (vi) Recognizing individuals in videos, etc. A sample set of face images of a specific individual is shown in Figure 1. Notice the extreme variations for a single person as described earlier.

The database along with annotations will be made available for research.

\section{Indian Movie Face Database}

We designed Indian Movie Face Database (IMFDB) database primarily as a benchmark for face recognition algorithms in unconstrained settings. IMFDB is built from frames extracted from Indian movies of different languages. IMFDB database consists of 34512 facial images corresponding to 100 Indian actors collected from approximately 103 movies. IMFDB consists of 67 male and 33 female actors with atleast 200 images for each actor. IMFDB comes with detailed annotation in terms of age, bounding box, movie release, expression, gender, pose, makeup, and possible kind of occlusion. The database is designed through following steps:

1) Selection of movies and actors,

2) Selection of frames from videos,

3) Cropping of faces,

4) Pruning the database and

5) Annotation

We describe each of these steps in the following subsections.

\section{A. Selection of movies and actors}

Identification of actors and movies became the critical part in designing the database and optimizing the human labor. First, in order to ensure the diversity in appearance, we selected the movies from 5 languages namely, Hindi,
Telugu, Kannada, Malayalam, and Bengali. All the movies are collected from personal collection and YouTube. In the second step, we selected the actors that have a long career span so that we can obtain multiple movies of the actors. For each actor, we selected the movies that give wide variations in age. For example, for Amitabh Bachchan who has a career span of 30 years, we collected frames from Anand and Kabhi Kushi Kabhi ghum which he acted in 1971 and 2001 respectively. As far as resolution and quality is concerned, old movies were at poorer resolution while new movies were available at different resolutions. This implicitly resulted in variation in terms of resolution and quality of images. The number of movies selected for each actor varied from $2-5$. For lead actors, it is easy to obtain large number of images from 2 movies while for supporting actors there are usually few frames per movie. Thus for supporting actors we usually collected the frames from $2-5$ movies.

Since the images are extracted through a manual process, it is important to minimize the number of movies as much as possible in order to reduce the manual labor. During this stage, we carefully selected the movies in such a way that there is a maximum overlap of actors across movies. For example, with 7 kannada movies Nagarahavu, Yejamana, Apthamithra, Odahuttidavaru, Bangaradmanusha, Bahaddur gandu, and Habba, we collected frames of 23 actors. For each actor, these movies gave all the variations including significant age variations. Note that, we did not compromise on quality of images (diverse variations) while selecting the movies and characters. In the final step, if the required number of frames are not available for an actor, we collected short movie clips of the actor from YouTube to reach minimum number of 200 .

\section{B. Selection of frames}

Once the movies are collected, we extracted the frames from these videos with frame interval of 10 . We observed that many faces which might pose a serious challenge to face recognition algorithms were not getting detected by the Viola-Jones face detector. So to build a database that offers serious challenge to recognition algorithms, we resorted to manual selection of frames. The immediate question that comes up is, which are the right frames to select? The answer may vary from person to person. However, we followed few heuristics, so that manual selection of frames by different people are consistent. First, we considered only one frame with signification variation from a shot unless there is another frame with significant difference with the first frame. Second, if there are multiple variations available in a shot, faces with occlusion and pose variation, which offer a serious challenge to recognition algorithms compared to facial expression and illumination are prefered. Third, we did not consider the frames with small faces and difficult to recognize manually. The minimum number of frames selected for each subject from different movies is 200. For lead actors who appear in majority of the frames, we collected more than 400 frames from different movies.

\section{Cropping of faces}

After the frames are selected, we manually cropped the faces with a tight bounding box. We used the matlab tool 

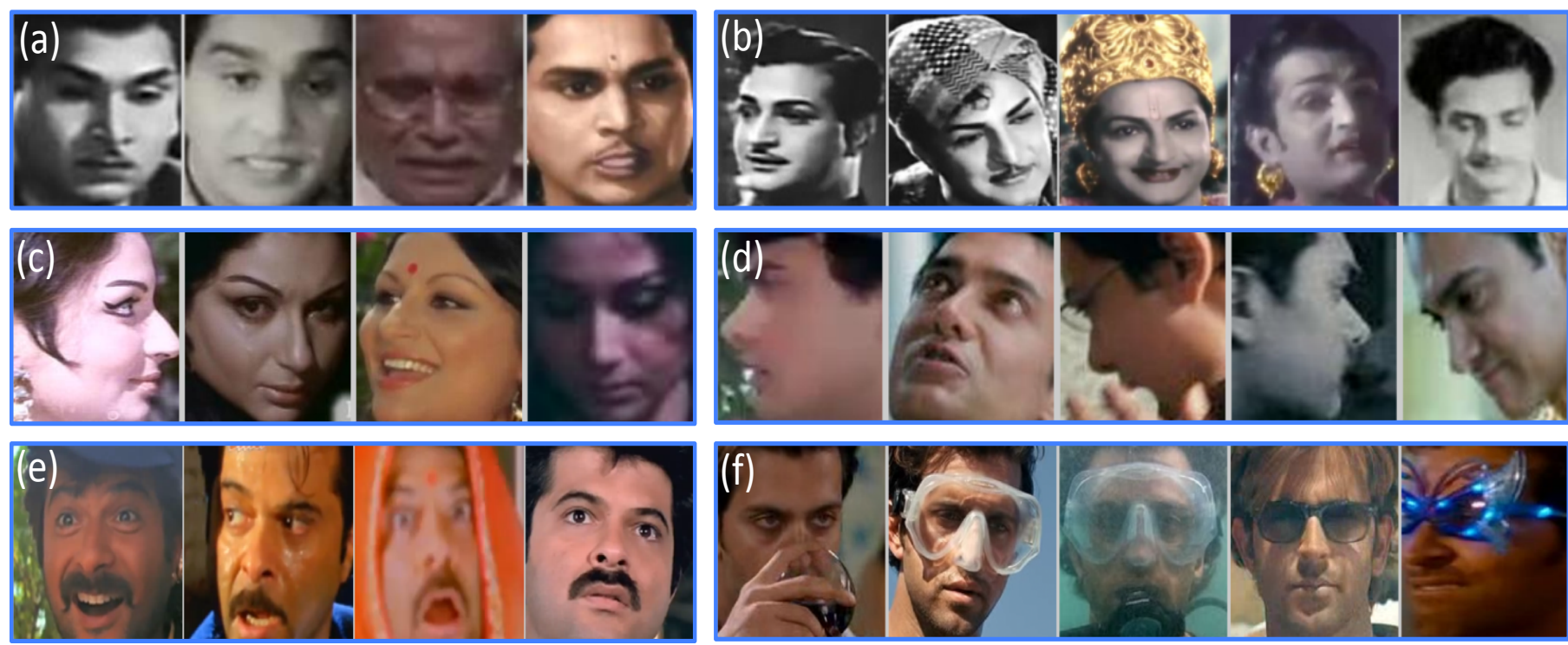

Figure 2. Widely varying appearance of faces based on (a) Age, (b) Make-up, (c) Illumination, (d) Pose, (e) Expressions and (f) Occlusions from IMFDB.

imcrop which gives the cropped region and bounding box information on selecting the face. It is difficult to define a bounding box for a face as it varies from pose to pose. Nevertheless, in order to maintain consistency across images, we followed a heuristic of cropping the face from forehead to chin.

\section{Pruning the database}

As a post-processing step, through a careful inspection we removed any duplicates or similar images for each subject. We must note that though we attempted to remove all duplicates and similar images, there may exist some similar images that were not found. We believe that the number of these instances is small enough so that they will not significantly impact research.

\section{MAnUAL ANNOTATION}

IMFDB provides a detailed annotation through a careful analysis of each and every image. We manually annotated the following attributes for each face:

- Expressions: Anger, Happiness, Sadness, Surprise, Fear, Disgust.

- Illumination: Bad, Medium, High.

- Pose: Frontal, Left, Right, Up, Down.

- Occlusion: Glasses, Beard, Ornaments, Hair, Hand, None, Others.

- Age: Child ( $1-12$ years), Young (13 - 30 years), Middle ( $31-50$ years), Old (Above 50 years).

- Makeup: Partial makeup, Over-makeup.

- Gender: Male, Female.

These attributes and annotations are motivated by the large variety of problems related to face recognition. Some of

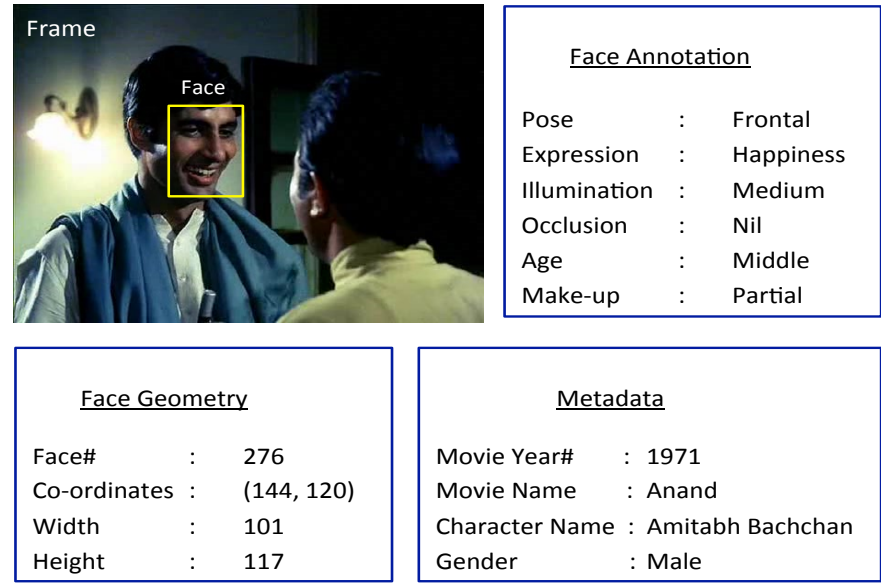

Figure 3. Face annotation, geometry and metadata of actor Amitabh Bachchan from the movie Anand.

them are subjective (e.g. expression) while some of them are objective (e.g. age, which is validated from facts about the actors and the release dates of the movies). Even though all possible set of values for certain attributes such as occlusion, pose, and expression could be quite large, we tried to annotate for most commonly occurring attribute values in the movies.

Figure 2 shows few examples from the IMFDB with different attributes in Indian movies. Notice the diversity among the examples. In addition to face annotation attributes (as stated above) for each face, geometric and metadata details such as movie information, bounding box, character information are also mentioned as shown in Figure 3.

The IMFDB consists of both gray scale and RGB face images. Figure 4 shows the face annotation details of sample faces from IMFDB exposing different facial expressions, pose variations, gender, make-up, partial occlusions, age and illumination conditions. 

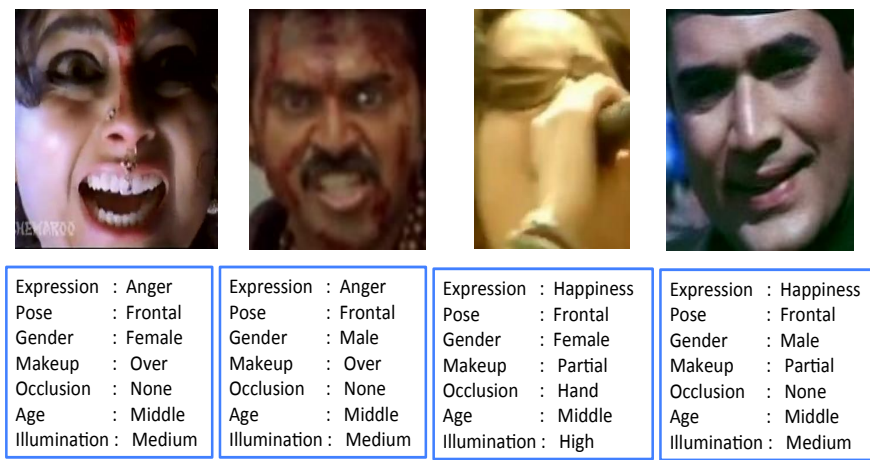

Figure 4. Examples from IMFDB showing face annotation details of Soundarya, Venkatesh, Katrina Kaif and Rajesh Khanna.

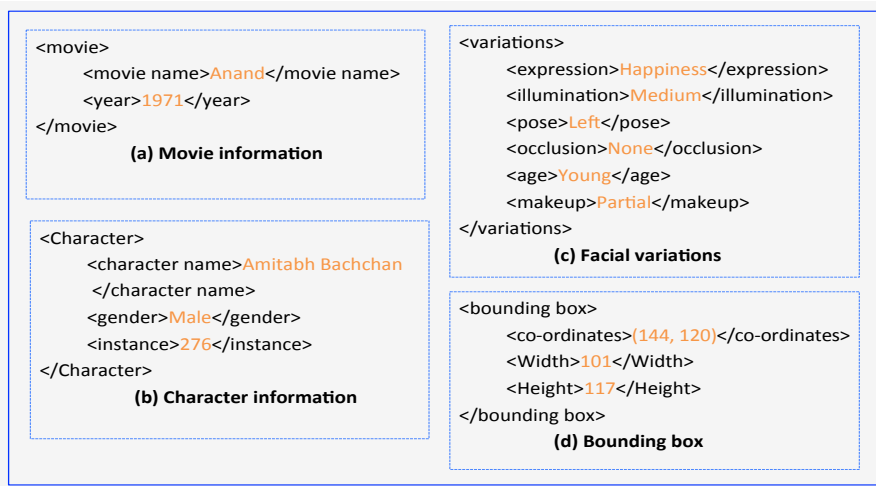

Figure 5. Sample XML specification for actor Amitabh Bachchan as shown in Figure 3.

Annotated information is stored as XML description. An XML specification consists of four main nested elements such as (a) Movie information consisting of movie name and year of its release (b) Character information specifying actor name, gender and instance number assigned to it (c) Facial variations specifying the type of facial expression, illumination, pose, occlusion, age and makeup, and (d) Bounding box consisting of $(x, y)$ co-ordinates of top left corner in the frame along with height and width of the cropped face. Figure 5 shows XML specification of a sample face considered in Figure 3. IMFDB is the first face database that provides a complete XML specification of annotation as opposed to most popular benchmarked databases.

Figure 6 shows a sample dataset of 100 face images, one image selected from each character of IMFDB. This gives a quick glimpse of all the Indian actors selected for constructing the database.

\section{DATABASE COMPLEXITY ANALYSIS}

In this section, we provide an analysis of the database that gives some insights about its complexity. IMFDB is designed with a careful observation in order to have a diverse range of variations for each subject. We have shown one such example in Figure 7 that shows large amount of variability for actor Vishnuvardhan.

A possible way to measure the complexity of the database is by understanding the distribution of faces. Ideally, database

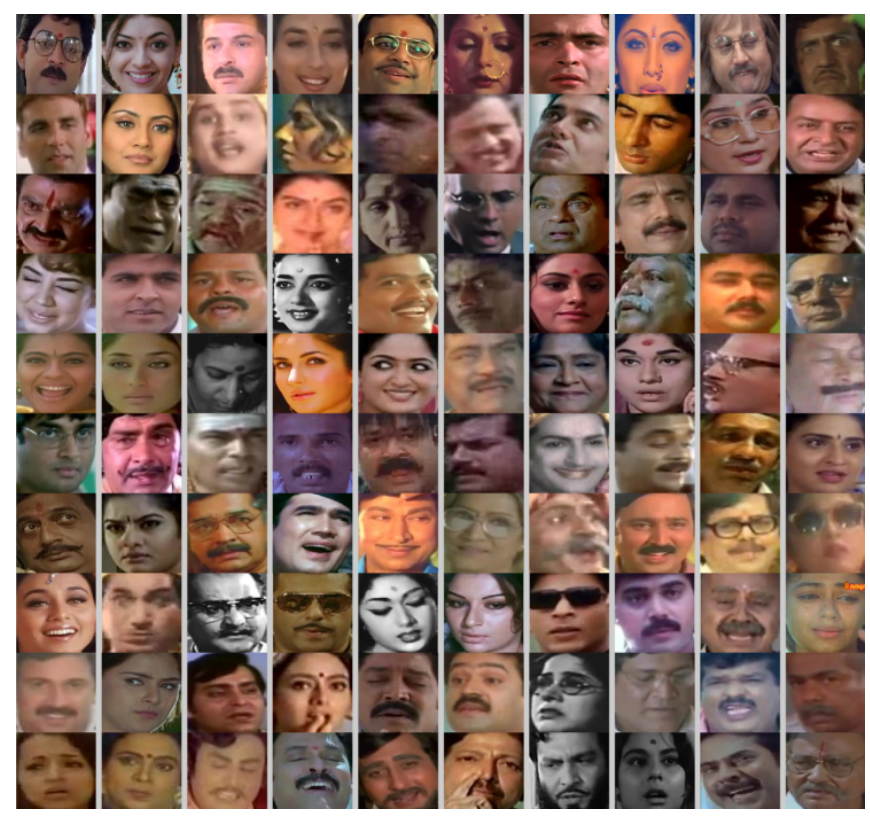

Figure 6. Sample faces of 100 character from IMFDB.

should have a broad distribution so that faces that are drawn from it have a diverse set of variations. For this experiment, we randomly selected a total of 500 images from 5 male actors (100 from each actor) from LFW, PubFigs and IMFDB. We analyzed the distribution of the faces by observing the eigen value distribution of covariance matrix of the selected faces. The magnitude of eigen values indicate the prominant directions along which data has maximum variance. If the database has less variations, then the number of prominant principal components required to summarize the data will be less. Figure 9 shows the eigen value spectrum of LFW, PubFigs and IMFDB. It is clear that, number of components required to span the space for IMFDB is large compared to LFW and PubFigs database. One of the reasons why LFW and PubFigs require less components is attributed to the possible number of poses imposed by a face detector. Also, there are not many images in LFW which occur under extreme lighting conditions, or very low lighting condition [8]. Figure 8 and Figure 10 shows the corresponding mean faces and eigen vectors for the same subset of databases. While the average face of IMFDB is more spreaded, it resembles the structure of face for LFW and PubFigs indicating less variations. This is attributed to limited pose variations constrained by the detector.

Finally, we conducted an experiment on faces of Indian movie actor Shahrukh Khan common between PubFigs and IMFDB. Figure 11 show the mean faces for the actors instances from PubFigs and IMFDB. The mean face of PubFigs has a close resemblance with the true appearance of the actor indicating less variations (especially pose) for the actor in the database.

\section{CONCLUSION}

In this paper, we have introduced a new database called IMFDB for face recognition research. It is developed with the intention of providing a common benchmark for face recognition and allied research. The main characteristics of the 


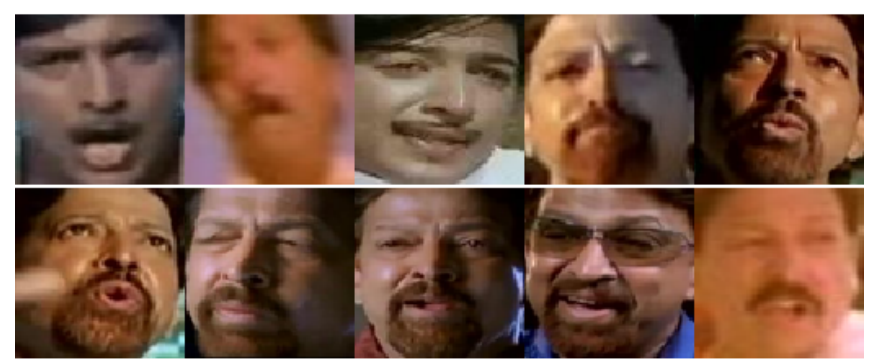

(a) Expression

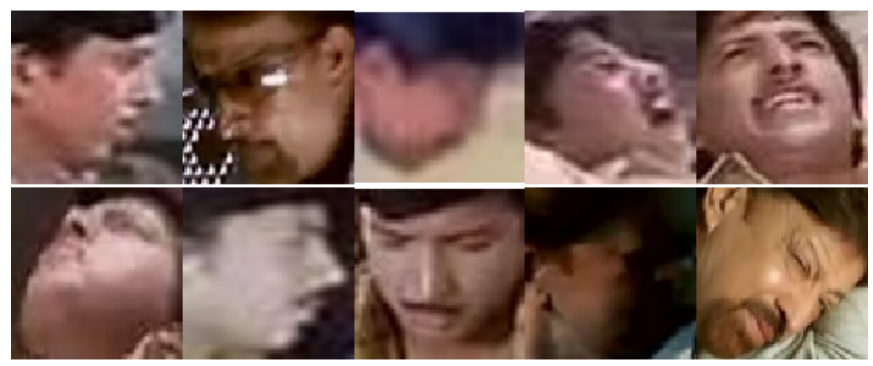

(b) Pose

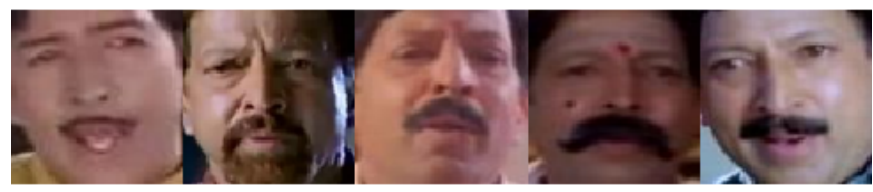

(c) Age

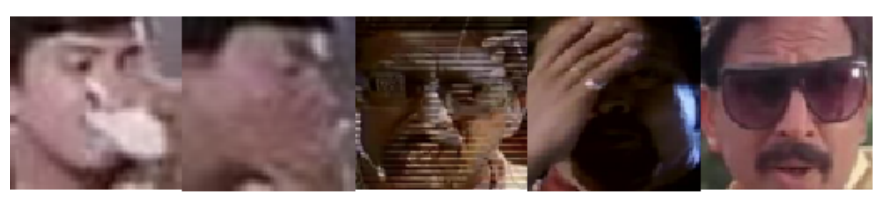

(d) Occlusion

Figure 7. Examples from IMFDB demonstrating large variablility for actor Vishnuvardhan in terms of (a) expression (b) Pose, (c) age and (d) occlusion.

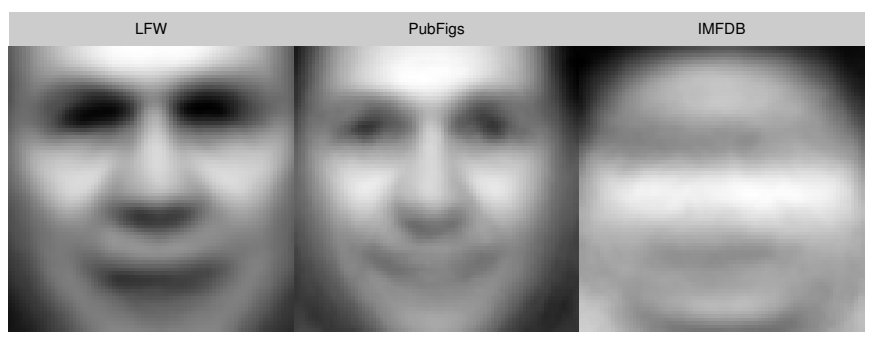

Figure 8. Mean faces of subset of databases with 5 male actors from (left) LFW, (middle) PubFigs and (right) IMFDB.

IMFDB face database are: 1) large number of images: 34512 face images from 100 subjects; 2) large diversity in terms of pose, age, expression, occlusion, illumination, make-up and the combined variations; 3) Manual selection of frames and bounding box and 4) a detailed manual XML annotation . By making this database available to the research community, we hope to encourage the exploration of many unsolved problems.

\section{ACKNOWLEDGEMENTS}

We would like to thank CVIT staff Phaneendra, Nandini, Bhadri, and Phani Kumar for their help in annotation.
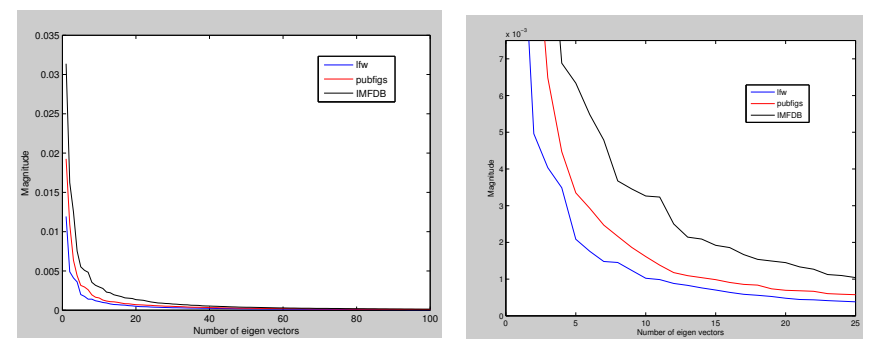

Figure 9. Eigen value spectrum of subset of LFW, PubFigs, and IMFDB of 5 male actors. It is zoomed in (right) for less than 25 eigen vectors.

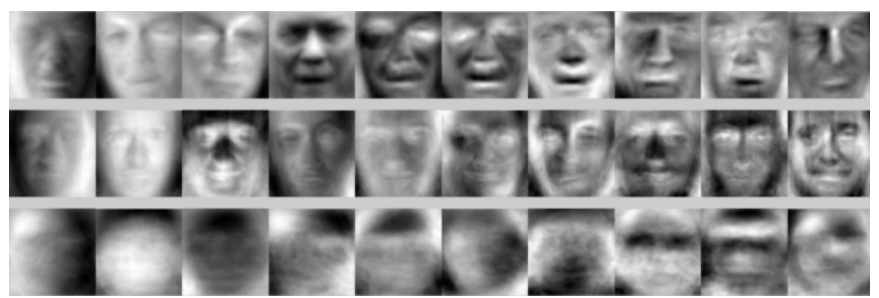

Figure 10. Top 10 eigen vectors of subset of (top) LFW, (middle) PubFigs, and (bottom) IMFDB of 5 male actors

\section{REFERENCES}

[1] W. Zhao Sarnoff, R. Chellappa, J. Phillips, and A. Rosenfeld, "Face recognition: A literature survey," in ACM Comput. Surv., 2003.

[2] A. S. Georghiades, P. N. Belhumeur, and D. J. Kriegman, "From few to many: Illumination cone models for face recognition under variable lighting and pose," in PAMI, 2001.

[3] A. Martínez and R. Benavente, "The AR face database," in $C V C$ Technical Report, 1998.

[4] D. Jelsovka, R. Hudec, and M. Breznan, "Face recognition on FERET face database using LDA and CCA methods," in IEEE TSP, 2011.

[5] T. Sim, S. Baker, and M. Bsat, "The CMU Pose, Illumination, and Expression Database," in PAMI, 2003.

[6] P. N. Belhumeur, J. P. Hespanha, and D. J. Kriegman, "Eigenfaces vs. Fisherfaces: Recognition Using Class Specific Linear Projection," in PAMI, 1997.

[7] J. Wright, A. Y. Yang, A. Ganesh, S. S. Sastry, and Y. Ma, "Robust Face Recognition via Sparse Representation," in PAMI, 2009.

[8] G. B. Huang, M. Ramesh, T. Berg, and E. Learned-Miller, "Labeled faces in the wild: A database for studying face recognition in unconstrained environments," University of Massachusetts, Amherst, Tech. Rep., 2007.

[9] N. Kumar, A. C. Berg, P. N. Belhumeur, and S. K. Nayar, "Attribute and simile classifiers for face verification," in ICCV, 2009.

[10] J. C. Klontz and A. K. Jain, "A Case Study on Unconstrained Facial Recognition using the Boston Marathon Bombings suspects," Michigan State University, Tech. Rep., 2013.

[11] V. Jain and A. Mukherjee, "The Indian face database," 2002. [Online]. Available: http://vis-www.cs.umass..edu/ vidit/IndianFaceDatabase/

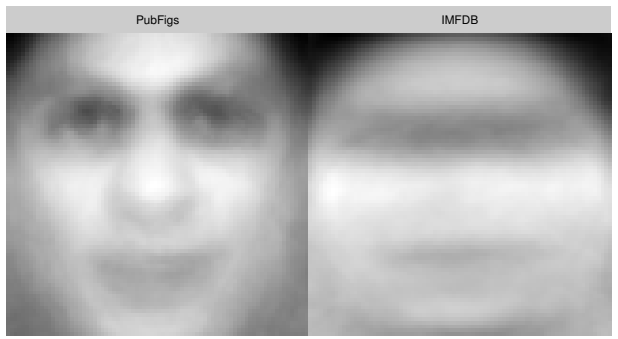

Figure 11. Mean face of PubFigs (left) and IMFDB (right) for Shahrukh Khan 